\title{
Haematological malignancies implications during the times of the COVID-19 pandemic (Review)
}

\author{
ELENI PAPAKONSTANTINOU ${ }^{1}$, KONSTANTINA DRAGOUMANI ${ }^{1}$, ASPASIA EFTHIMIADOU $^{2}$, \\ ANASTASIA MARINA PALAIOGEORGOU ${ }^{1}$, KATERINA PIEROULI ${ }^{1}$, THANASIS MITSIS ${ }^{1}$, \\ GEORGE P. CHROUSOS $^{3,4}$, FLORA BACOPOULOU ${ }^{3}$ and DIMITRIOS VLACHAKIS ${ }^{1,3,4}$ \\ ${ }^{1}$ Laboratory of Genetics, Department of Biotechnology, School of Applied Biology and Biotechnology, \\ Agricultural University of Athens, 11855 Athens; ${ }^{2}$ Hellenic Agricultural Organization-Demeter, Institute of \\ Soil and Water Resources, Department of Soil Science of Athens, 14123 Lycovrisi; ${ }^{3}$ University Research Institute \\ of Maternal and Child Health and Precision Medicine, and UNESCO Chair on Adolescent Health Care, \\ National and Kapodistrian University of Athens, Aghia Sophia Children's Hospital; ${ }^{4}$ Division of Endocrinology \\ and Metabolism, Center of Clinical, Experimental Surgery and Translational Research, \\ Biomedical Research Foundation of the Academy of Athens, 11527 Athens, Greece
}

Received August 18,2021; Accepted October 18, 2021

DOI: $10.3892 / 01.2021 .13117$

\begin{abstract}
The COVID-19 pandemic has complicated current healthcare services for cancer patients. Patients with haematological malignancies specifically seem vulnerable to SARS-CoV-2 infection due to their immunosuppressed status. The COVID-19 pandemic influences every step of the assessment and treatment of a haematological malignancy. Clinicians must adhere to strict policies to not spread the virus to their patients while they must also adjust their workflow for maximum productivity. These difficulties accentuate the ever-present need to improve the healthcare services for cancer patients. This improvement is needed not only to combat the problems that arose from the COVID-19 pandemic but also to establish a framework for the management of patients with haematological malignancies in potential future pandemics.
\end{abstract}

Correspondence to: Dr Dimitrios Vlachakis, Laboratory of Genetics, Department of Biotechnology, School of Applied Biology and Biotechnology, Agricultural University of Athens, 75 Iera Odos, 11855 Athens, Greece

E-mail:dimvl@aua.gr

Dr Flora Bacopoulou, University Research Institute of Maternal and Child Health and Precision Medicine, and UNESCO Chair on Adolescent Health Care, National and Kapodistrian University of Athens, Aghia Sophia Children's Hospital, 8 Thivon and Livadias Street, 11527 Athens, Greece

E-mail: fbacopoulou@med.uoa.gr

Key words: haematological, malignancies, cancer, COVID-19, pandemic, translational genetics, healthcare services, SARS-CoV-2

\section{Contents}

1. Introduction

2. Haematological malignancies and healthcare services

3. The COVID-19 pandemic and healthcare services

4. Haematological malignancies and COVID-19: Current obstacles

5. Discussion and conclusions

\section{Introduction}

Haematological malignancies refer to a distinct group of cancers that affect the bone marrow, blood and lymphatic systems (1). Some of the most well-studied haematological malignancies include leukaemia, non-Hodgkin lymphomas, Hodgkin lymphoma, multiple myelomas, myelodysplastic syndromes and myeloproliferative neoplasms $(2,3)$. These types of cancer, along with other haematology-related diseases, contribute substantially to global mortality and morbidity (4). Haematological malignancies are some of the most commonly occurring cancers and are a leading cause of cancer-related mortality, particularly for older adults who display grim survival rates $(5,6)$. These patients present with significant immune deficits due to intense therapeutic procedures, with infections being a frequent problem, leading to higher mortality and morbidity and more frequent, prolonged and expensive hospitalization (7). Patients with haematological malignancies are therefore considered vulnerable when it comes to healthcare provision and hospitalization.

In December 2019, a pneumonia outbreak of unknown origins was reported in Wuhan City in Hubei Province in central China (8). These outbreaks were later found to be due to a novel virus that belongs to the family Coronoviridae, called SARS-CoV-2, with the associated disease being called Coronavirus Disease 2019 (COVID-19) (9). COVID-19 
infection was officially declared as a pandemic by the World Health Organization on March 11th, 2020 (10). According to epidemiological data, COVID-19 is a more deadly disease than influenza (11). COVID-19 is an infectious disease of the respiratory system characterized mainly by a severe respiratory distress syndrome combined with additional symptoms, including fever, dry cough, body aches, fatigue, diarrhoea, conjunctivitis, hypoxia, central cyanosis, shock, coma, convulsions, sepsis, prolonged immobilization, dyspnoea and pneumonia (12-15). The main processes responsible for the multi-organ damage leading to death from COVID-19 include hyperinflammation, hypercoagulability and severe hypoxemia. The first process refers to the disruption of the immune system whose cells penetrate and affect various organs, and which, in the case of this virus, causes abnormal activation of T cells and macrophages that leads to a 'storm of cytokines' causing hyperinflammation. The second process involves the formation of blood clots due to the ordered activation and functioning of the immune system, which leads to endothelial damage and increased blood clotting. The third process is a result of the previous two processes, where due to the abundance of cytokines and blood clots in the pulmonary circulation, reduced and insufficient oxygenation is observed (16). SARS-CoV-2 can spread through droplets and human-to-human transmission (direct contact), and through contaminated objects and airborne contagion (indirect contact) (17). Severe cases of COVID-19 are frequent in elderly patients and individuals already affected by other pathologies (18). COVID-19 has become a global threat to healthcare systems with cumulatively 102.1 million cases and over 2.2 million deaths reported as of January 31st, 2021 (19). From January 2021, $\sim 10$ vaccines have received emergency authorization for use in humans (20). Although promising, these vaccines cannot lead to an immediate return to the normality of the pre-COVID world (21-23). Several governments have implemented stringent social distancing measures and lockdowns in an effort to reduce the probability of people contracting the virus $(24,25)$. This new reality has greatly affected people's lives, increasing the feeling of fear and stress on an individual level but also causing economic recession, job loss and stigmatization on a social level $(11,26)$, altogether leading to the increase in addictive issues, including alcohol consumption. According to the literature, lockdowns combined with insecurity and fear can lead to increased alcohol consumption during quarantine; however, alcohol is a factor that affects and weakens the immune system, contributes to obesity and mental disorders and increases the risk of contracting diseases, including COVID-19 (26). Furthermore, the quarantine measures have excluded patients with chronic diseases, such as cancer, from institutional health care settings, leading to a negative effect on mental health and emotional well-being (27).

During the COVID-19 pandemic, patients with haematological malignancies display an increased risk of mortality due to COVID-19 infection while their mental health can also be influenced negatively (28-30). It is therefore essential to develop efficient policies to manage patients with haematological malignancies during not only the COVID-19 pandemic but also potential future pandemics (Fig. 1). Healthcare providers need to overcome specific challenges such as timely completion of the diagnostic and staging studies, thus providing treatment to patients without exposing themself to infection, monitoring patient health status, and even identifying potential mental health issues $(27,31)$. The new healthcare policies must provide the right treatment to the right patient, in the right place and at the right time through the use of modern technology to overcome these difficulties (32).

The provision of such treatment can be achieved through precision medicine (33). Precision medicine allows clinicians to make accurate diagnoses and provide targeted therapies for patients according to their disease's biological background and accounting for individual differences in their genetic makeup, environment, behaviours, lifestyle and cultures (34). The development of cost-effective high-throughput analysis of biological molecules has allowed the generation and processing of large biological datasets (omics data), which can be used for the development of precision medicine (35). Translational genetics uses information produced from such technologies, specifically genomic information, to develop new healthcare strategies and clinical applications $(36,37)$. The present literature review aims to describe how translational genetics may help developing better healthcare strategies for patients with haematological malignancies under pandemic situations such as COVID-19.

\section{Haematological malignancies and healthcare services}

A haematological malignancy diagnosis ranges from a chronic, slow-progressing condition to a fast growing, frequently fatal disease (38). An early cancer diagnosis is essential since it improves the patient's prognosis (39). The diagnosis of these types of cancer is quite complex and achieved through histology, cytology, cytogenetics, immunophenotype and imaging (40). These methods exhibit high cost and complexity while also being highly time-consuming (41). Furthermore, patients themselves might complicate diagnosis based on their ability to understand their symptoms and react accordingly (42). Therefore, early diagnosis provides numerous challenges for both patients and clinicians. Patients should determine when to seek help and clinicians should identify potential symptoms and make accurate and timely referrals to secondary care (43). It is imperative to improve current methodologies in haematological malignancies diagnosis for better and more timely healthcare services. At present, because genetics serves an essential role in the diagnosis of most haematological malignancies, the ever-advancing genetic technologies may provide additional techniques to ameliorate the diagnosis of these types of cancer (44).

The most commonly used measures to determine cancer patients' prognosis and the disease trajectory are survival statistics (45); however, patients with haematological malignancies are confronted to an unpredictable illness trajectory where the possibility of cure persists even in relapsed and refractory periods (46). In addition, prognostication becomes even more difficult since deterioration is oftentimes so unpredictable and rapid that it may lead to abrupt changes in healthcare goals (47). A prime example is that due to their immunocompromised status and potential sepsis, patients are highly likely to be admitted to an intensive care unit (ICU), where their prognosis worsens significantly (48). Cytogenetics have truly helped improving the prognostics of haematological 
COVID-19 and management of patients with haematological

malignancies

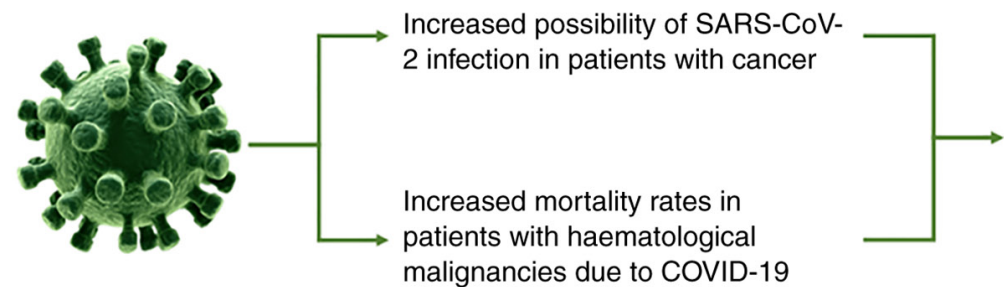

malignancies due to COVID-19

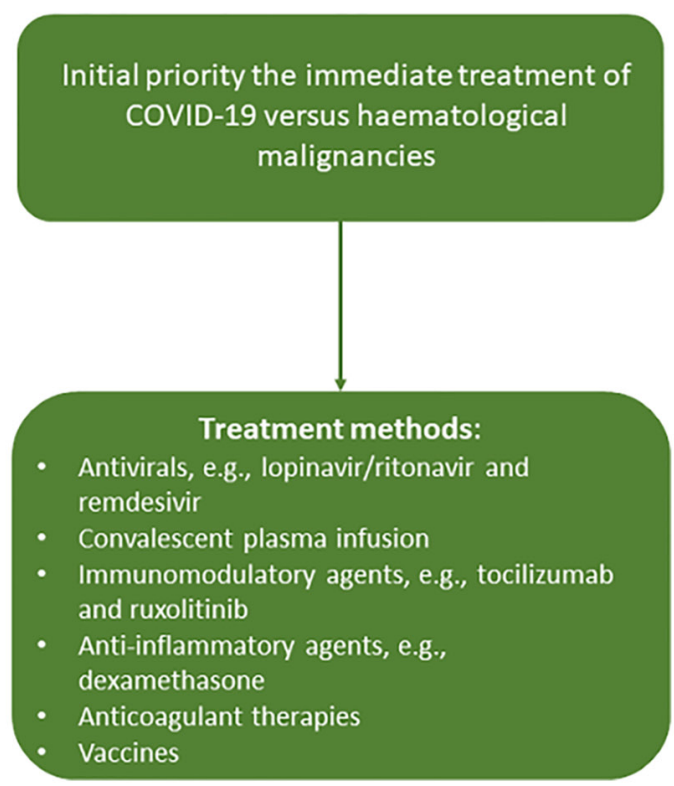

Figure 1. COVID-19 and management of patients with haematological malignancies.

malignancies, where based on a patient's genetic background, a clinician may better evaluate the disease's trajectory (3). In particular, genomics has identified numerous genetic loci that may influence a haematological malignancy prognosis, while modern technological advances may elucidate additional genetic loci of interest.

Each subtype of haematological malignancy is unique and treatment is therefore based on several factors, including certain biomarkers, the patient's clinicopathological characteristics and stage of the disease. Some commonly used therapeutic approaches include chemotherapy and autologous stem-cell transplantation (49). Advancements in the field of immunology regarding haematological malignancies have helped developing numerous immunotherapies, such as conventional monoclonal antibodies, bispecific T-cell engagers and therapeutic molecules that block inhibitory T-cell pathway (50). Important advances have therefore been made in the field of therapeutics; however, some difficulties exist, which are mainly caused by the high molecular diversity and complexity of these diseases, the variable evolution during disease and therapy, and the development of chemoresistance and immune evasion mechanisms (51). A better knowledge of the molecular mechanisms governing the pathogenesis, development, metabolism, metastasis and relapse of cancer, and the determination of similarities and differences across distinct haematological malignancies, may help optimizing current treatments or provide adjacent and alternative therapeutics (51). Modern advances in genomic research can help with such a task.

In the case of cancer, precision medicine aims to improve treatment efficiency for patients, using genomic information from each patient's tumour. Genetic biomarkers can differ significantly depending on the type of cancers, making disease prediction a complex process. Through new generation technologies, including Whole Genome Sequencing or Exome Sequencing, a variety of molecular biomarkers and targets have now been determined and comprise cancer-related DNA and RNA changes, such as small nucleotide variants, epigenetic modifications (DNA methylation, fusion genes, copy number alterations, mutational signature patterns) and gene expression level modifications (3). Epigenetic marks, which mainly involve DNA methylation, histone modifications and chromatin remodelling, can be triggered by a response to external stimuli and occur throughout life in healthy tissues, and play a key role in the onset and development of numerous complex human diseases, such as cancer, autoimmune disorders, neurodegenerative diseases, obesity and psychiatric disorders (52).

Haematological malignancies are pathological conditions where the possibility for pharmacological manipulation and therapeutic targeting through drugs that could act on DNA methylation reactions exists (53). In previous studies where large-scale sequencing of cancer genomes was performed, various mutations in enzymes that regulate DNA methylation in haematological malignancies were identified. For example, in acute myelogenous leukaemia (AML), $44 \%$ of patients develop mutations in genes responsible for regulating genital DNA methylation, such as the DNMT3A methyltransferase genes, isocitrate dehydrogenase 1 and 2 (IDH1 and IDH2) and TET oncogene family member 2 (TET2) (54-56). In addition, TET 2 mutations are common in angioimmunoblastic $\mathrm{T}$ cell lymphoma (AITL), which is one of the most common subtypes of peripheral T cell lymphoma, as well as IDH2 mutations that have been reported in 20-45\% of AITL cases $(57,58)$.

In general, cancer treatment using epigenetic regulators is highly valuable in haematological malignancies. The US Food and Drug Administration (FDA) has already approved some DNA demethylating agents, such as decitabine and 5-azacitidine, for cases of myelodysplasia and AML (53). Another class 
of drugs that act as epigenetic regulators comprise the inhibitors of histone deacetylases (HDACs). Two HDAC inhibitors that have also been approved by the FDA are vorinostat and romidepsin, which are used in some cases of haematological malignancies (59). Furthermore, it has been reported that the combination of drugs targeting DNA methylation and histone modifications could be beneficial in the treatment of haematological malignancies (60).

\section{The COVID-19 pandemic and healthcare services}

COVID-19 is a particularly life-threatening virus in patients with haematological malignancies. According to initial reports, cancer patients are estimated to be twice as likely to be contaminated with SARS-CoV-2 compared with the general population, and in case of infection, these patients are at higher risk of serious episodes, leading to ICU, invasive ventilation or death, compared with patients without cancer $(61,62)$. Since patients with haematological malignancies, such as leukaemia, lymphoma, myeloma, and recipients of autologous or allogeneic haematopoietic stem cell transplantation, show significant humoral and cellular immunosuppression, their infection with COVID-19 leads to significant higher mortality rates. According to recent studies, the overall mortality associated with COVID-19 is $32-40 \%$ in patients with haematological malignancies $(63,64)$. It is therefore crucial to identify some prognostic factors that could help assessing the mortality risks of these patients.

Patients with haematological malignancies infected by SARS-CoV-2 require special protocols followed by medical professionals. The guideline followed in severe cases includes as an initial priority the immediate treatment of COVID-19 vs. haematological malignancies. Generally, due to severe immunosuppression, patients should be isolated and the treatment applied should be proportional to the severity of the virus infection (65).

To directly target the virus in multiple organs, the use of antivirals, such as lopinavir/ritonavir and remdesivir, as well as antibodies, are being evaluated for safety and appropriateness for the management of COVID-19 $(66,67)$. According to results from previous studies, convalescent plasma infusion has exhibited more favourable results in improving symptoms and biomedical indicators, thus proving greater safety and benefits compared with other drugs $(68,69)$. However, as mentioned before, infection with SARS-CoV-2 initiates a storm of cytokines that can cause several side effects, including hypercoagulable inflammatory condition, increased risk of secondary infections and weakening of the immune system (70). Subsequently, certain drugs are used to limit cytokine storms, such as targeted immunomodulatory agents, including tocilizumab and ruxolitinib, and anti-inflammatory agents, including dexamethasone. Thrombosis prevention is also crucial among patients with COVID-19. For this purpose, low molecular weight heparins are the first treatment of choice, although other anticoagulant therapies, such as antithrombin, can also be used (71).

Between the end of 2020 and the beginning of 2021, newly developed vaccines against SARS-COV-2 were available. Vaccine development is generally time and cost consuming, with an average development time of 10.7 years. Under normal conditions, new vaccines are tested in vivo in animals and then in humans through clinical trials. However, the immediate need to develop anti-SARS-COV-2 vaccines led to a new process where the animal stage was bypassed (72). SARS-COV-2 vaccination aims to prevent symptomatic or severe infection that would require hospitalization, prevent transmission of the virus and generate a strong immune response against viral protein $S$, thus preventing the virus from attaching to human cells (23). At present, two mRNA vaccines and two adenovirus vaccines have been developed and distributed in the European Union. The side effects of these vaccines are limited to local injection site reactions, such as edema and erythema, which may be followed by non-specific systemic effects, such as fever, myalgia, chills, fatigue and headache, although these are considered to be the results of the immune system reaction occurring after vaccination. Very rarely, severe allergic reactions have been reported, resulting in the exclusion of individuals with a history of severe allergic reactions to any component of the vaccine. However, data availability on the safety of these vaccines in terms of interaction with other vaccines or drugs, use during pregnancy or breast-feeding, and in vulnerable subgroups, such as cancer patients, including haematological malignancies, patients with diabetes, neurological diseases and with autoimmune or inflammatory disorders, is still deficient, although the benefits of the vaccines are likely to outweigh the risks of vaccine-related adverse reactions. Investigating the safety of vaccines in these cases is therefore crucial necessary $(73,74)$.

\section{Haematological malignancies and COVID-19: current obstacles}

The risk of severe outcome from COVID-19 increases with advancing age and the existence of comorbidities (75). Cancer patients require therefore additional attention due to their immunosuppressed status, which is a result of both anti-cancer treatment and tumour growth (76). Furthermore, some complications of COVID-19 comprise hypercoagulability and thrombosis, and cancer patients already display an elevated risk of venous thromboembolic events (77). In particular, cancer patients have been shown to exhibit a higher risk of severe COVID-19, ICU admission and mechanical ventilation (78). Oncology societies around the world have developed some guidelines to treat patients without risking a potential COVID-19 infection. These guidelines categorize patients into different priorities based on the severity of their cancer condition and if postponing healthcare services has an impact on overall survival or quality of life (75). It is important to note that numerous additional factors are crucial for the clinician's approach to cancer patients during a pandemic, including cancer type, cancer therapy type, existing comorbidities, COVID-19 treatment and the capacity of the healthcare system itself (78).

The above considerations apply to haematologic cancer patients, with certain unique characteristics of these diseases complicating the relationship between COVID-19 and cancer. Most haematologic cancer patients receive anti-cancer drugs that suppress bone marrow function or display cancers of the immune system, and present therefore with an even higher 
risk of community and hospital-acquired infections (79). Furthermore, it has been reported that patients with haematological malignancies and COVID-19 display significantly higher rates of severe disease and mortality compared with COVID-19 cases in the general population (76). These observations suggest that COVID-19 infection may result in increased mortality in patients with haematological malignancies (77).

Since patients with haematological malignancies display a particularly high risk for COVID-19, they should be considered a high priority group for SARS-CoV-2 vaccination (80). However, widespread vaccination of high-risk patients is a time-consuming procedure while the COVID-19 pandemic is not slowing down (81). In addition, the use of anti-COVID-19 drugs in such patients should be previously evaluated and heavily monitored since potential drug-drug interactions with anti-cancer drugs or unexpected toxicity of anti-COVID-19 drugs could emerge $(77,82)$. At present, prevention policies still remain the most important part when it comes to combating COVID-19. Both clinicians and patients with haematological malignancies should adhere to a strict set of guidelines to prevent spreading of COVID-19 (83). These guidelines may include creating designated areas for patients at high-risk for COVID-19 infection, following specific cleaning and social distancing practices, having virtual meetings instead of requiring patients to attend the hospital, and screening a large number of patients and clinicians. Furthermore, the effects of COVID-19 on patients with haematological malignancies will be long-lasting due to delays in cancer scientific study, which is influenced by restrictions on research activities and the diversion to COVID-19 research funding $(76,81)$.

\section{Discussion and conclusions}

Translational genetics, which is the application of genetic knowledge to improve healthcare and disease prevention, can provide the basis for more advanced healthcare services that could alleviate problems arising from pandemics. The best use of translational genetics information would be to tailor therapies to each individual. This field of precision medicine can provide novel approaches for the diagnosis, prognosis, and treatment of individuals with haematological malignancies. Through this personalization of healthcare services, clinicians can improve their efficiency and help managing diseases such as haematological malignancies under difficult circumstances like those of a pandemic.

The present review was limited to the amount of existing data regarding patients suffering from haematological malignancies, as well as to the existing data concerning SARS-CoV-2 and COVID-19. As the pandemic progresses, novel aspects of COVID-19 infection and its effect on patients with cancer and treatment strategies may arise. However, this review explored the complex interaction between COVID-19 and haematological cancer in patients, describing the obstacles and proposing strategies to overcome them. Similar studies examining cancer and COVID-19 implications on various levels have been published, although they do not particularly focus on haematological malignancies $(84,85)$. In addition, the impact of
COVID-19 on haematological malignancy patients has been described by a recent review, although it is based on survey data and is limited to data from one single country (86). It is therefore crucial to further investigate the impact of COVID-19 on haematological malignancies, and evaluate their consequent treatment with specialized medication and adjusted hospitalization requirements.

\section{Acknowledgements}

Not applicable.

\section{Funding}

This research was supported by the European and Developing Countries Clinical Trials Partnership (EDCTP2) under the Horizon 2020 'Research and Innovation Actions' DESCA (grant no. RIA2018EF-2081), and the European Regional Development Fund of the European Union and Greek national funds through the Operational Program Competitiveness, Entrepreneurship and Innovation, under the call RESEARCH-CREATE-INNOVATE (grant no. T2EDK-02222).

\section{Availability of data and materials}

Not applicable.

\section{Authors' contributions}

EP, KD, AE, AMP, KP, TM, GPC, FB and DV contributed to conceptualization, design, writing, drafting, revising, editing and reviewing of the manuscript. All authors have read and approved the final manuscript. Data authentication is not applicable.

\section{Ethics approval and consent to participate}

Not applicable.

\section{Patient consent for publication}

Not applicable.

\section{Competing interests}

The authors declare that they have no competing interests.

\section{References}

1. Pearce L: Haematological cancers. Nurs Stand 30: 15, 2016.

2. Pérez GB, Calaf GM, Villalba MTM, Prieto KS and Burgos FC: Frequency of hematologic malignancies in the population of Arica, Chile. Oncol Lett 18: 5637-5643, 2019.

3. Taylor J, Xiao W and Abdel-Wahab O: Diagnosis and classification of hematologic malignancies on the basis of genetics. Blood 130: 410-423, 2017.

4. The Lancet Haematology: The global burden of haematological diseases. Lancet Haematol 5: e1, 2018.

5. Krok-Schoen JL, Fisher JL, Stephens JA, Mims A, Ayyappan S, Woyach JA and Rosko AE: Incidence and survival of hematological cancers among adults ages $\geq 75$ years. Cancer Med 7: 3425-3433, 2018. 
6. Egesie OJ, Agaba PA, Silas OA, Achenbach C, Zoakah A Agbaji OO, Madaki JA, Okeke EN, Hou L, Sagay AS and Murphy R: Presentation and survival in patients with hematologic malignancies in Jos, Nigeria: A retrospective cohort analysis. J Med Trop 20: 49-56, 2018.

7. Khayr W, Haddad RY and Noor SA: Infections in hematological malignancies. Dis Mon 58: 239-249, 2012.

8. Harapan H, Itoh N, Yufika A, Winardi W, Keam S, Te H, Megawati D, Hayati Z, Wagner AL and Mudatsir M: Coronavirus disease 2019 (COVID-19): A literature review. J Infect Public Health 13: 667-673, 2020.

9. Esakandari H, Nabi-Afjadi M, Fakkari-Afjadi J, Farahmandian N, Miresmaeili SM and Bahreini E: A comprehensive review of COVID-19 characteristics. Biol Proced Online 22: 19, 2020.

10. Muralidar S, Ambi SV, Sekaran S and Krishnan UM: The emergence of COVID-19 as a global pandemic: Understanding the epidemiology, immune response and potential therapeutic targets of SARS-CoV-2. Biochimie 179: 85-100, 2020.

11. Neagu M, Calina D, Docea AO, Constantin C, Filippini T, Vinceti M, Drakoulis N, Poulas K, Nikolouzakis TK, Spandidos DA and Tsatsakis A: Back to basics in COVID-19: Antigens and antibodies-completing the puzzle. J Cell Mo Med 25: 4523-4533, 2021

12. Sarkar C, Mondal M, Torequl Islam M, Martorell M, Docea AO, Maroyi A, Sharifi-Rad J and Calina D: Potential therapeutic options for COVID-19: Current status, challenges, and future perspectives. Front Pharmacol 11: 572870,2020

13. Kostoff RN, Kanduc D, Porter AL, Shoenfeld Y, Calina D, Briggs MB, Spandidos DA and Tsatsakis A: Vaccine- and natural infection-induced mechanisms that could modulate vaccine safety. Toxicol Rep 7: 1448-1458, 2020.

14. Rudrapal M, Khairnar SJ, Borse LB and Jadhav AG: Coronavirus disease-2019 (COVID-19): An updated review. Drug Res (Stuttg) 70: 389-400, 2020

15. Calina D, Hernández AF, Hartung T, Egorov AM, Izotov BN, Nikolouzakis TK, Tsatsakis A, Vlachoyiannopoulos PG and Docea AO: Challenges and scientific prospects of the newest generation of mRNA-based vaccines against SARS-CoV-2. Life (Basel) 11: 907, 2021

16. Islam MT, Salehi B, Karampelas O, Sharifi-Rad J, Docea AO, Martorell $\mathrm{M}$ and Calina D: High skin melanin content, vitamin D deficiency and immunity: Potential interference for severity of COVID-19. Farmacia 68: 970-983, 2020.

17. Lotfi M, Hamblin MR and Rezaei N: COVID-19: Transmission, prevention, and potential therapeutic opportunities. Clin Chim Acta 508: 254-266, 2020

18. Giammaria D and Pajewski A: Can early treatment of patients with risk factors contribute to managing the COVID-19 pandemic? J Glob Health 10: 010377, 2020.

19. WHO: Weekly epidemiological update-2 February 2021. Journal 2021.

20. Lee ACK and Morling JR: COVID-19 vaccine dilemmas. Public Health, 2021

21. Badiani AA, Patel JA, Ziolkowski K and Nielsen FBH: Pfizer: The miracle vaccine for COVID-19? Public Health Pract (Oxf) 1: 100061,2020

22. Calina D, Docea AO, Petrakis D, Egorov AM Ishmukhametov AA, Gabibov AG, Shtilman MI, Kostoff R, Carvalho F, Vinceti M, et al: Towards effective COVID-19 vaccines: Updates, perspectives and challenges (review). Int J Mol Med 46: 3-16, 2020.

23. Calina D, Sarkar C, Arsene AL, Salehi B, Docea AO, Mondal M, Islam MT, Zali A and Sharifi-Rad J: Recent advances, approaches and challenges in targeting pathways for potential COVID-19 vaccines development. Immunol Res 68 : 315-324, 2020.

24. Alfano V and Ercolano S: The efficacy of lockdown against COVID-19: A cross-country panel analysis. Appl Health Econ Health Policy 18: 509-517, 2020.

25. Papakonstantinou E, Pierouli K, Mitsis T, Diakou KI Palaiogeorgou AM, Bacopoulou F, Chrousos GP, Eliopoulos E and Vlachak is D: COVID-19 global social lockdowns: Energy-related, psychological, epigenetic, health and environmental impacts (review). Int J Epigen 1: 8, 2021.

26. Calina D, Hartung T, Mardare I, Mitroi M, Poulas K, Tsatsakis A, Rogoveanu I and Docea AO: COVID-19 pandemic and alcohol consumption: Impacts and interconnections. Toxicol Rep 8: 529-535, 2021.
27. Moraliyage H, De Silva D, Ranasinghe W, Adikari A, Alahakoon D, Prasad R, Lawrentschuk N and Bolton D: Cancer in lockdown: Impact of the COVID-19 pandemic on patients with cancer. Oncologist 26: e342-e344, 2020.

28. Rubinstein SM and Warner JL: COVID-19 and haematological malignancy: Navigating a narrow strait. Lancet Haematol 7: e701-e703, 2020

29. Wang Y, Duan Z, Ma Z, Mao Y, Li X, Wilson A, Qin H, Ou J, Peng K, Zhou F, et al: Epidemiology of mental health problems among patients with cancer during COVID-19 pandemic. Transl Psychiatry 10: 263, 2020.

30. Islam MT, Hossen M, Kamaz Z, Zali A, Kumar M, Docea AO, Arsene AL, Călina D and Sharifi-Rad J: The role of hmgbl in the immune response to sars-cov-2 infection: From pathogenesis towards a new potential therapeutic target. Farmacia 69: 621-634, 2021.

31. Isidori A, de Leval L, Gergis U, Musto P and Porcu P: Management of patients with hematologic malignancies during the COVID-19 pandemic: Practical considerations and lessons to be learned. Front Oncol 10: 1439, 2020.

32. Binder AF, Handley NR, Wilde L, Palmisiano N and Lopez AM: Treating hematologic malignancies during a pandemic: Utilizing telehealth and digital technology to optimize care. Front Oncol 10: 1183, 2020

33. Jørgensen JT: Twenty years with personalized medicine: Past, present, and future of individualized pharmacotherapy. Oncologist 24: e432-e440, 2019.

34. Divaris K: Fundamentals of precision medicine. Compend Contin Educ Dent 38 (8 Suppl): S30-S32, 2017.

35. Manzoni C, Kia DA, Vandrovcova J, Hardy J, Wood NW, Lewis PA and Ferrari R: Genome, transcriptome and proteome: The rise of omics data and their integration in biomedical sciences. Brief Bioinform 19: 286-302, 2018.

36. D'Souza RN, Dunnwald M, Frazier-Bowers S, Polverini PJ, Wright JT, de Rouen T and Vieira AR; Translational Genetics Meeting Group: Translational genetics: Advancing fronts for craniofacial health. J Dent Res 92: 1058-1064, 2013.

37. Vlachakis D and Kossida S: Antibody drug conjugate bioinformatics: Drug delivery through the letterbox. Comput Math Methods Med 2013: 282398, 2013.

38. Geres $\mathrm{H}$ and Kotchetkov R: Nursing in patients with hematological malignancies. Int J Hematol Oncol 9: IJH24, 2020.

39. Vedsted P and Olesen F: Early diagnosis of cancer-the role of general practice. Scand J Prim Health Care 27: 193-194, 2009.

40. Li J, Smith A, Crouch S, Oliver S and Roman E: Estimating the prevalence of hematological malignancies and precursor conditions using data from haematological malignancy research network (HMRN). Cancer Causes Control 27: 1019-1026, 2016.

41. Wang Y, Zhang B, Zhang X, Wang X, Cheng J and Chen B Detection and identification of hematologic malignancies and solid tumors by an electrochemical technique. PLoS One 11: e0153821, 2016.

42. Dapkevičiūtė A, Šapoka V, Martynova E and Pečeliūnas V: Time from symptom onset to diagnosis and treatment among haematological malignancies: Influencing factors and associated negative outcomes. Medicina (Kaunas) 55: 238, 2019.

43. Howell DA, Smith AG, Jack A, Patmore R, Macleod U, Mironska E and Roman E: Time-to-diagnosis and symptoms of myeloma, lymphomas and leukaemias: A report from the haematological malignancy research network. BMC Hematol 13: 9, 2013.

44. Prakash G, Kaur A, Malhotra P, Khadwal A, Sharma P, Suri V, Varma N and Varma S: Current role of genetics in hematologic malignancies. Indian J Hematol Blood Transfus 32: 18-31, 2016.

45. Mariotto AB, Noone AM, Howlader N, Cho H, Keel GE Garshell J, Woloshin S and Schwartz LM: Cancer survival: An overview of measures, uses, and interpretation. J Natl Cancer Inst Monogr 2014: 145-186, 2014

46. Gray TF, Temel JS and El-Jawahri A: Illness and prognostic understanding in patients with hematologic malignancies. Blood Rev 45: 100692, 2021

47. Button E, Chan RJ, Chambers S, Butler J and Yates P: A systematic review of prognostic factors at the end of life for people with a hematological malignancy. BMC Cancer 17: 213, 2017.

48. de Vries VA, Müller MCA, Arbous MS, Biemond BJ, Blijlevens NMA, Kusadasi N, Span LRF, Vlaar APJ, van Westerloo DJ, Kluin-Nelemans HC, et al: Long-term outcome of patients with a hematologic malignancy and multiple organ failure admitted at the intensive care. Crit Care Med 47: e120-e128, 2019 
49. Kansara RR and Speziali C: Immunotherapy in hematologic malignancies. Curr Oncol 27 (Suppl 2): S124-S131, 2020.

50. Nelson MH and Paulos CM: Novel immunotherapies for hematologic malignancies. Immunol Rev 263: 90-105, 2015.

51. Méndez-Ferrer S, Bonnet D, Steensma DP, Hasserjian RP, Ghobrial IM, Gribben JG, Andreeff $M$ and Krause DS: Bone marrow niches in haematological malignancies. Nat Rev Cancer 20: 285-298, 2020.

52. Boland MJ, Nazor KL and Loring JF: Epigenetic regulation of pluripotency and differentiation. Circ Res 115: 311-324, 2014

53. Tsai HC, Li H, Van Neste L, Cai Y, Robert C, Rassool FV, Shin JJ, Harbom KM, Beaty R, Pappou E, et al: Transient low doses of DNA-demethylating agents exert durable antitumor effects on hematological and epithelial tumor cells. Cancer Cell 21: 430-446, 2012.

54. Im AP, Sehgal AR, Carroll MP, Smith BD, Tefferi A, Johnson DE and Boyiadzis M: DNMT3A and IDH mutations in acute myeloid leukemia and other myeloid malignancies: Associations with prognosis and potential treatment strategies. Leukemia 28 : $1774-1783,2014$.

55. Li KK, Luo LF, Shen Y, Xu J, Chen Z and Chen SJ: DNA methyltransferases in hematologic malignancies. Semin Hematol 50: 48-60, 2013.

56. Wakita S, Yamaguchi H, Omori I, Terada K, Ueda T, Manabe E, Kurosawa S, Iida S, Ibaraki T, Sato Y, et al: Mutations of the epigenetics-modifying gene (DNMT3a, TET2, IDH1/2) at diagnosis may induce FLT3-ITD at relapse in de novo acute myeloid leukemia. Leukemia 27: 1044-1052, 2013

57. Cairns RA, Iqbal J, Lemonnier F, Kucuk C, de Leval L, Jais JP Parrens M, Martin A, Xerri L, Brousset P, et al: IDH2 mutations are frequent in angioimmunoblastic T-cell lymphoma. Blood 119: 1901-1903, 2012.

58. Lemonnier F, Couronné L, Parrens M, Jaïs JP, Travert M, Lamant L, Tournillac O, Rousset T, Fabiani B, Cairns RA, et al: Recurrent TET2 mutations in peripheral T-cell lymphomas correlate with TFH-like features and adverse clinical parameters. Blood 120: 1466-1469, 2012

59. Azad N, Zahnow CA, Rudin CM and Baylin SB: The future of epigenetic therapy in solid tumours-lessons from the past. Nat Rev Clin Oncol 10: 256-266, 2013.

60. Cheng Y, He C, Wang M, Ma X, Mo F, Yang S, Han J and Wei X: Targeting epigenetic regulators for cancer therapy: Mechanisms and advances in clinical trials. Signal Transduct Target Ther 4 : 62, 2019.

61. Dai M, Liu D, Liu M, Zhou F, Li G, Chen Z, Zhang Z, You H, Wu M, Zheng Q, et al: Patients with cancer appear more vulnerable to SARS-CoV-2: A multicenter study during the COVID-19 outbreak. Cancer Discov 10: 783-791, 2020.

62. Vijenthira A, Gong IY, Fox TA, Booth S, Cook G, Fattizzo B, Martín-Moro F, Razanamahery J, Riches JC, Zwicker J, et al: Outcomes of patients with hematologic malignancies and COVID-19: A systematic review and meta-analysis of 3377 patients. Blood 136: 2881-2892, 2020

63. MalardF, Genthon A, BrissotE, van de WyngaertZ, Marjanovic Z, Ikhlef S, Banet A, Lapusan S, Sestilli S, Corre E, et al: COVID-19 outcomes in patients with hematologic disease. Bone Marrow Transplant 55: 2180-2184, 2020.

64. Wang B, Van Oekelen O, Mouhieddine TH, Del Valle DM, Richter J, Cho HJ, Richard S, Chari A, Gnjatic S, Merad M, et al: A tertiary center experience of multiple myeloma patients with COVID-19: Lessons learned and the path forward. J Hematol Oncol 13: 94, 2020.

65. Wang LQ, Tan Su Yin E, Wei GQ, Hu YX, Nagler A and Huang $\mathrm{H}$ : Weathering the storm: COVID-19 infection in patients with hematological malignancies. J Zhejiang Univ Sci B 21 921-939, 2020

66. Wiersinga WJ, Rhodes A, Cheng AC, Peacock SJ and Prescott HC: Pathophysiology, transmission, diagnosis, and treatment of coronavirus disease 2019 (COVID-19): A review. JAMA 324: 782-793, 2020.

67. Vlachakis D, Papakonstantinou E, Mitsis T, Pierouli K, Diakou I, Chrousos G and Bacopoulou F: Molecular mechanisms of the novel coronavirus SARS-CoV-2 and potential anti-COVID19 pharmacological targets since the outbreak of the pandemic. Food Chem Toxicol 146: 111805, 2020.

68. Psaltopoulou T, Sergentanis TN, Pappa V, Politou M, Terpos E, Tsiodras S, Pavlakis GN and Dimopoulos MA: The emerging role of convalescent plasma in the treatment of COVID-19. Hemasphere 4: e409, 2020.
69. Liu STH,Lin HM, Baine I, Wajnberg A, Gumprecht JP, Rahman F, Rodriguez D, Tandon P, Bassily-Marcus A, Bander J, et al: Convalescent plasma treatment of severe COVID-19: A propensity score-matched control study. Nat Med 26: 1708-1713, 2020.

70. Jamilloux Y, Henry T, Belot A, Viel S, Fauter M, El Jammal T, Walzer T, François B and Sève P: Should we stimulate or suppress immune responses in COVID-19? Cytokine and anti-cytokine interventions. Autoimmun Rev 19: 102567, 2020

71. Terpos E, Ntanasis-Stathopoulos I, Elalamy I, Kastritis E, Sergentanis TN, Politou M, Psaltopoulou T, Gerotziafas G and Dimopoulos MA: Hematological findings and complications of COVID-19. Am J Hematol 95: 834-847, 2020

72. Calina D, Hartung T, Docea AO, Spandidos DA, Egorov AM, Shtilman MI, Carvalho F and Tsatsakis A: COVID-19 vaccines: Ethical framework concerning human challenge studies. Daru 28: 807-812, 2020

73. Hernández AF, Calina D, Poulas K, Docea AO and Tsatsakis AM: Safety of COVID-19 vaccines administered in the EU: Should we be concerned? Toxicol Rep 8: 871-879, 2021.

74. Desai A, Gainor JF, Hegde A, Schram AM, Curigliano G, Pal S, Liu SV, Halmos B, Groisberg R, Grande E, et al: COVID-19 vaccine guidance for patients with cancer participating in oncology clinical trials. Nat Rev Clin Oncol 18: 313-319, 2021.

75. Gosain R, Abdou Y, Singh A, Rana N, Puzanov I and Ernstoff MS: COVID-19 and cancer: A comprehensive review. Curr Oncol Rep 22: 53, 2020.

76. García-Suárez J, de la Cruz J, Cedillo Á, Llamas P, Duarte R, Jiménez-Yuste V, Hernández-Rivas J, Gil-Manso R, Kwon M, Sánchez-Godoy $\mathrm{P}$, et al: Impact of hematologic malignancy and type of cancer therapy on COVID-19 severity and mortality: Lessons from a large population-based registry study. J Hematol Oncol 13: 133, 2020.

77. Wood WA, Neuberg DS, Thompson JC, Tallman MS, Sekeres MA, Sehn LH, Anderson KC, Goldberg AD, Pennell NA, Niemeyer CM, et al: Outcomes of patients with hematologic malignancies and COVID-19: A report from the ASH research collaborative data Hub. Blood Adv 4: 5966-5975, 2020.

78. ElGohary GM, Hashmi S, Styczynski J, Kharfan-Dabaja MA, Alblooshi RM, de la Cámara R, Mohmed S, Alshaibani A, Cesaro S, Abd El-Aziz N, et al: The risk and prognosis of COVID-19 infection in cancer patients: A systematic review and meta-analysis. Hematol Oncol Stem Cell Ther, Jul 30, 2020 (Epub ahead of print).

79. He W, Chen L, Chen L, Yuan G, Fang Y, Chen W, Wu D, Liang B, Lu X, Ma Y, et al: COVID-19 in persons with haematological cancers. Leukemia 34: 1637-1645, 2020.

80. Kuderer NM, Hill JA, Carpenter PA and Lyman GH: Challenges and opportunities for COVID-19 vaccines in patients with cancer. Cancer Invest 39: 205-213, 2021.

81. Luker GD and Boettcher AN: Impact of COVID-19 on clinical care and research in cancer imaging: Where we are now. Radiol Imaging Cancer 3: e210003, 2021.

82. Vlachakis D, Fakourelis P, Megalooikonomou V, Makris C and Kossida S: DrugOn: A fully integrated pharmacophore modeling and structure optimization toolkit. PeerJ 3: e725, 2015.

83. Fong D, San Nicolò KO, Alber M and Mitterer M: Evaluating the longitudinal effectiveness of preventive measures against COVID-19 and seroprevalence of IgG antibodies to SARS-CoV-2 in cancer outpatients and healthcare workers. Wien Klin Wochenschr 133: 359-363, 2021.

84. Cavalcanti IDL and Soares JCS: Impact of COVID-19 on cancer patients: A review. Asia Pac J Clin Oncol 17: 186-192, 2021

85. Zhang L, Zhu F, Xie L, Wang C, Wang J, Chen R, Jia P, Guan HQ, Peng L, Chen Y, et al: Clinical characteristics of COVID-19-infected cancer patients: A retrospective case study in three hospitals within Wuhan, China. Ann Oncol 31: 894-901, 2020.

86. Levy I, Sharf G, Norman S and Tadmor T: The impact of COVID-19 on patients with hematological malignancies: The mixed-method analysis of an Israeli national survey. Support Care Cancer: 1-9, 2021 (Epub ahead of print).

This work is licensed under a Creative Commons Attribution-NonCommercial-NoDerivatives 4.0 International (CC BY-NC-ND 4.0) License. 